\section{Regards sur l'économie allemande}

Bulletin économique du CIRAC

112 | 2014

Varia

\title{
Karlsruhe : la TV ZDF n'est pas assez indépendante
}

Isabelle Bourgeois

\section{OpenEdition}

\section{Journals}

Édition électronique

URL : http://journals.openedition.org/rea/4661

DOI : $10.4000 /$ rea.4661

ISBN : 978-2-8218-0826-3

ISSN : 1965-0787

Éditeur

CIRAC

Édition imprimée

Date de publication : 28 avril 2014

Pagination : 36-38

ISSN : 1156-8992

Référence électronique

Isabelle Bourgeois, «Karlsruhe : la TV ZDF n'est pas assez indépendante », Regards sur l'économie allemande [En ligne], 112 I avril 2014, mis en ligne le 16 mai 2014, consulté le 15 septembre 2020. URL : http://journals.openedition.org/rea/4661

Ce document a été généré automatiquement le 15 septembre 2020

(C) CIRAC 


\title{
Karlsruhe : la TV ZDF n'est pas assez indépendante
}

\author{
Isabelle Bourgeois
}

1 Dans un arrêt rendu le 25 mars 2014 ( 1 BvF 1/11 et 1 BvF 4/11), le Tribunal constitutionnel fédéral a affiné encore plus son abondante jurisprudence sur l'indépendance de l'audiovisuel public par rapport à l'Etat. En l'occurrence, il considère que les deux organes de contrôle de l'établissement de radiodiffusion commun aux 16 Länder qu'est la " Deuxième Télévision allemande », ZDF (Zweites Deutsches Fernsehen, siège: Mayence), comptent une part trop importante de personnalités politiques ou proches de l'Etat. Les 16 Länder disposent jusqu'au 30 juin 2015 pour réviser en ce sens le Contrat d'Etat portant création de cet établissement. Les Länder sont en effet seuls souverains en matière d'audiovisuel.

2 La Cour de Karlsruhe avait été saisie d'un contrôle en constitutionnalité par les Länder de Hambourg et de Rhénanie-Palatinat à la suite de la non-reconduction du rédacteur en chef de la ZDF, Klaus Brender, en 2009. Alors que le président de cet établissement souhaitait prolonger son contrat, certains membres du conseil d'administration s'étaient ligués sous la houlette du ministre-président de Hesse (Roland Koch, CDU) pour s'y opposer. Cette "affaire Brender " avait à l'époque fait couler beaucoup d'encre, confirmant ce que nombre d'experts pointaient depuis longtemps, à savoir la non-conformité des statuts de la ZDF avec le principe constitutionnel de l'indépendance vis-à-vis de l'Etat, corollaire de la liberté des médias garantie par l'art. 5 de la Loi fondamentale.

\section{Sur la sellette : la composition} des organes de contrôle interne

3 Les établissements de radiodiffusion de droit public allemands (dont la ZDF) se distinguent de France Télévision ou de Radio France sur un certain nombre de points. Il s'agit de personnes morales bénéficiant de la capacité juridique ; elles sont donc dotées de l'autonomie financière et de la responsabilité administrative. Indépendants de l'Etat, ces établissements sont une émanation de la collectivité des citoyens qui les a chargés 
d'une mission de service public et qui les contrôle seule. Ils ont donc une structure spécifique permettant l'exercice du contrôle interne par la société civile. Chaque établissement a ainsi trois organes : l'Intendant (président) qui dirige l'établissement, en est le représentant légal et en porte la responsabilité éditoriale. La ligne éditoriale est définie par le conseil de surveillance (nommé Fernsehrat : conseil de la TV dans le cas de la ZDF) qui exerce également le contrôle des programmes; ce conseil, qui élit l' Intendant, incarne le contrôle par la société et est composé de représentants de la société civile organisée (partenaires sociaux, Eglises, associations...) et d'un nombre restreint de représentants du monde politique (députés, membres de partis politiques). Enfin, la gestion de l'établissement est contrôlée par le conseil d'administration, composé majoritairement de membres de l'exécutif des Länder.

ZDF : nette

politisation des organes

4 Dans le cas de la ZDF - le seul établissement créé conjointement par tous les Länder, ce qui implique nombre de compromis -, cette politisation est poussée à l'extrême : le conseil d'administration (14 membres) compte 5 représentants des gouvernements des Länder et un du gouvernement fédéral ; ses huit autres membres sont nommés par le Fernsehrat. Celui-ci, qui compte 77 membres (c'est de loin le conseil de surveillance le plus important en nombre dans l'audiovisuel allemand), comprend $44 \%$ de personnalités politiques.

\section{Indépendance de l'Etat}

et pluralisme interne

permettent au maximum $33 \%$

de personnalités politiques

5 C'est beaucoup trop, vient de rappeler la Cour de Karlsruhe. Le maximum compatible avec le principe constitutionnel d'indépendance vis-à-vis de l'Etat et avec le pluralisme de l'information comme des programmes qu'exige sa mission de service public, est d'un tiers. En effet, l'obligation faite aux législateurs par l'art. 5, \$1 al. 2 de la Loi fondamentale de garantir la liberté de la radiodiffusion "vise à la création d'un ordre [organisation; IB] garantissant que le pluralisme des opinions existantes puisse s'exprimer dans toute sa diversité et en totalité dans l'audiovisuel ». Si l'organisation concrète (statuts, composition des organes...) relève de la liberté du législateur, il doit respecter impérativement "la contribution au pluralisme des contenus qui incombe au service public et que le seul marché ne peut garantir». La condition sine qua non de ce pluralisme éditorial est le respect de la diversité des opinions existantes et de leur représentation au sein des instances décisionnelles.

6 L'impératif de la garantie du pluralisme concrétise donc l'impératif de l'indépendance vis-à-vis de l'Etat. En clair : les organes doivent être "configurés de manière à rendre effectivement impossible une prise d'influence de l'information par des acteurs politiques de l'Etat ou proches de l'Etat pour faire valoir des intérêts particuliers ou des agendas partisans, tout particulièrement ceux des partis politiques». Non seulement le nombre de personnalités politiques dans les instances doit donc être limité, mais également leur influence: "le législateur doit tout mettre en ceuvre pour éviter la domination d'approches majoritaires dans l'opinion comme la pétrification de la composition des instances». A cet effet, 
tous les membres des deux conseils doivent pouvoir agir selon leur seule conscience ; il leur est donc interdit de respecter des consignes, quelles qu'elles soient. Enfin, les comptes rendus des séances doivent être rendus publics, dans la mesure du possible en temps réel, par voie de publication ou de communiqué de presse par exemple.

\section{Un arrêt dans le droit fil de celui « sur la TV Adenauer » de 1961}

7 C'est une ironie du sort que cet arrêt soit rendu à propos de la ZDF. Cet établissement avait en effet vu le jour en juin 1961 - à la suite du premier arrêt rendu par Karlsruhe sur l'audiovisuel, le 28 février 1961. Ce «Jugement sur la télévision Adenauer », dans lequel la Cour définissait pour la première fois l'indépendance de l'Etat découlant directement de l'art. 5 de la Loi fondamentale est, depuis, la 'grande charte' garantissant l'indépendance de tous les médias - vis-à-vis de l'Etat comme de tout intérêt particulier privé. A la fin des années 1950, le chancelier Adenauer, considérant que les radios et télévisions existantes (celles de l'ARD) diffusaient des programmes un peu trop 'de gauche' à son goût, avait lancé la création de ce qu'en droit français, on appellerait une société nationale de programmes : une chaine appartenant à l'Etat et sous sa tutelle. Ce projet a été déclaré contraire à la Constitution et, depuis, l'exécutif allemand est constitutionnellement incompétent en matière d'audiovisuel; seuls les législateurs des Länder sont habilités à adopter des lois sur leur audiovisuel ou à conclure des contrats d'Etat entre eux si une affaire dépasse les compétences ou les intérêts d'un seul Land. Reste le problème que ces contrats d'Etat doivent être signés par les ministres-présidents des Länder, autrement dit leurs chefs de gouvernement...

L'arrêt de 2014 sur la ZDF sera lourd de conséquences pour les structures de l'ensemble de l'audiovisuel public allemand: des établissements membres de l'ARD en passant par Deutschlandradio et Deutsche Welle jusqu'aux autorités de régulation du secteur privé (les 'CSA' des Länder) dont les instances présentent une structure analogue. Car la ZDF n'est pas le seul établissement à présenter une politisation de ses instances. C'est aussi le cas par exemple de la télévision bavaroise (Bayerischer Rundfunk), de celle des Länder de Saxe, Saxe-Anhalt et Thuringe (Mitteldeutscher Rundfunk) ou de Radio Bremen. Ces établissements membres de l'ARD (groupement de travail des radios et TV des Länder) et les six autres ont en tout cas décidé préventivement de se pencher sur la composition de leurs organes internes lors de la réunion de leurs dirigeants le 30 avril. Et, selon le président de leur conférence permanente, Uwe Grund, l'arrêt de Karlsruhe devrait avoir pour conséquence « une harmonisation des lois sur l'audiovisuel sur les grandes questions de principe » (epd medien, 09-04-2014). Les Länder ont 14 mois pour réformer les structures de contrôle interne de l'audiovisuel, c'est-à-dire pour "revoir les 25 lois" afférentes (V. Henle, Revue européenne des médias, n 30/2014). Connaissant les rivalités historiques et les intérêts politiques en jeu, on peut affirmer sans crainte que ce sera là une rude tâche. Mais le fonctionnement de la démocratie allemande y gagnera encore en transparence comme en pertinence. (IB) 
INDEX

Mots-clés : télévision, politique, tribunal, constitution

\section{AUTEUR}

\section{ISABELLE BOURGEOIS}

Chargée de recherche au CIRAC et rédactrice en chef deRegards sur l'économie allemande 